\title{
Osteosíntesis percutánea con tornillo mini-Acutrak en las fracturas agudas de escafoides
}

\author{
C. Lamas ${ }^{(1)}$, I. Proubasta ${ }^{(2)}$, E. Moya ${ }^{(3)}$, O. Buezo ${ }^{(3)}$, S. Cortés ${ }^{(3)}$, \\ J. Colomina ${ }^{(3)}$ \\ Hospital de la Santa CReu i Sant Pau. Universidad Autónoma de Barcelona \\ (1) MÉdico AdJunto de la UNIDAD DE LA MANO Y EXTREMIDAD SUPERIOR. \\ Diploma de CiRUGía de la MANO DE LA FESSH. SERVICIO DE COT

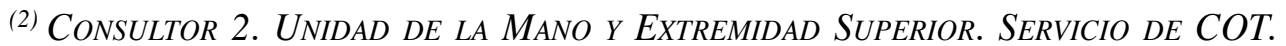 \\ PROFESOR ASOCIADO DE LA UNIVERSIDAD AUTÓNOMA DE BARCELONA \\ (3) MÉdico InTERNo RESIDENTE DE COT
}

\author{
Correspondencia: \\ Dra. Claudia Lamas \\ Unidad de Mano y Extremidad Superior \\ Departamento de Cirugía Ortopédica y Traumatología \\ Hospital de la Santa Creu i Sant Pau \\ Universidad Autónoma de Barcelona \\ C/ Sant Antoni M. Claret, 167 \\ 08025 Barcelona \\ Teléfono: 34935537031 \\ Fax: 34935537033 \\ Correo electrónico: clamasg@hsp.santpau.es
}

Objetivos. Evaluamos nuestra experiencia con el tornillo canulado percutáneo mini-Acutrak en el tratamiento de las fracturas agudas de escafoides.

Material y Método. Desde noviembre de 2007 a septiembre del 2008 realizamos un estudio retrospectivo en 22 pacientes con fractura de escafoides. El patrón de fractura se dividió según la clasificación de Herbert y por los criterios de inestabilidad propuestos por Cooney et al. No se incluyen las fracturas de cuerpo de escafoides inestables. Las fracturas mínimamente o no desplazadas de cuerpo de escafoides se trataron con un tornillo percutáneo vía palmar. En las fracturas de polo proximal y oblicuas de cuerpo se colocó el tornillo vía dorsal. Posterior a la osteosíntesis se realiza una inmovilización de 10 días y seguidamente fisioterapia.

Resultados. De los 22 pacientes intervenidos uno fue una mujer y 21 fueron varones de edad media 27 años (rango: 18-47). El seguimiento medio fue de 6 meses (rango: 3-10). La osteosíntesis percutánea se indicó en 15 fracturas mínimamente o no
Purpose. We had evaluated our experience with mini-Acutrak percutaneous cannulated screw fixation of acute scaphoid fractures.

Materials and Methods. From november 2007 to september 2008, 22 patients were operated for traumatic injuries in scaphoid. Scaphoid fractures have been classified by Herbert. We have also followed the criteria proposed by Cooney et al. We have not included unstable waist fractures. A transverse waist fracture is ideally suited to stable fixation with screw placed from the volar direction. In proximal pole and oblique fractures the cannulated screws were placed dorsally. After percutaneous screw fixation was placed into the scaphoid 10 days of immobilization and early active motion of the wrist joint is allowed.

Results. There were 1 female and 21 male with mean age 27 (range: 18-47). The follow-up was a mean of 6 months (range: 3-10). Percutaneous screw fixation was indicated for 15 minimally or non-displaced scaphoid waist fractures (Herbert type A2 or B2), 3 oblique fractures (Herbert type 
desplazadas de cuerpo escafoides (Herbert tipo A2 o B2), 3 fracturas oblicuas (Herbert tipo B1) y 4 de polo proximal (Herbert tipo B3). Todos los pacientes obtuvieron resultados buenos/excelentes con un tiempo de curación entre las 8 y 12 semanas (media de 6 semanas) y con un arco de movilidad completo. La tomografía axial computerizada fue útil en 8 casos para confirmar la consolidación. La longitud media de los tornillos fue de $18 \mathrm{~mm}$ (rango: 16-22). Como complicación tuvimos un caso de ruptura de la aguja guía en el interior del tornillo. No hemos apreciado casos de seudoartrosis, inestabilidad, infección, aflojamiento del tornillo ni cambios degenerativos.

Conclusiones. El tornillo de compresión percutáneo puede evitar el abordaje y los posibles daños de la osteosíntesis abierta. Si se realiza una indicación y vía de entrada correcta puede, en las fracturas agudas de escafoides mínimamente o no desplazadas, permitir la consolidación de la fractura con un tiempo de inmovilización menor y una incorporación precoz a las actividades habituales.

Palabras clave: fractura de escafoides, osteosíntesis escafoides, tornillo canulado, tornillo percutáneo, mini-Acutrak.
B1) and 4 proximal pole (Herbert type B3). All patients had good/excellent results with healing time to union ranged between 8 and 12 weeks (mean 6 weeks) and complete arc of motion. CT scan also may be useful in 8 cases to confirm the scaphoid union. Screw lengths were a mean of $18 \mathrm{~mm}$ (range: 16-22). In one patient the guide wire broke inside the cannulated implant. We had not seen nonunion, instability, infection, screw loosening or degenerative changes.

Conclusions. A percutaneously placed compression screw would avoid the potential pitfalls of open exposure of the scaphoid fractures and allow for earlier motion and rehabilitation. Percutaneous fixed nondisplaced scaphoid fractures had shorter time to union and time to return to work.

Key words: scaphoid fractures, scaphoid fixation, cannulated screw, percutaneous screw, mini-Acutrak.

Rev. Iberam. Cir. Mano - Vol. 36 • Núm. 2 Noviembre 2008 (88-94)

\section{INTRODUCCIÓN}

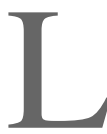

as fracturas agudas e inestables de escafoides carpiano se tratan mediante reducción y osteosíntesis abierta con tornillo de compresión. Las fracturas agudas y estables, sin embargo, normalmente se tratan aplicando una inmovilización con yeso de 6 a 8 semanas ${ }^{1}$. A pesar de una inmovilización apropiada, muchas fracturas de escafoides tienen un riesgo de no consolidación del $5 \%$ al $55 \%$, y otras consolidan en mala posición dando lugar a dolor en la muñeca secundaria a artrosis $^{2}$. En las fracturas de polo proximal se suma el hecho de que sin una osteosíntesis adecuada existe un riesgo elevado de necrosis avascular ${ }^{1,3}$.

La fractura de escafoides suele darse en una población de pacientes jóvenes con una alta demanda funcional y deportiva ${ }^{2}$. Para reducir el período de inmovilización en este grupo de pacientes, disminuir la rigidez de la muñeca y la pérdida de la actividad deportiva o laboral ${ }^{4}$, algunos autores utilizan el tornillo canulado percutáneo en las fracturas agudas de escafoides $^{2-7}$. Hasta la introducción de los tornillos percutáneos, todas las fracturas de escafoides quirúrgicas se trataban mediante un abordaje dorsal o palmar según el patrón de fractura, con la apertura de los ligamentos radio carpianos palmares o con lesión de estructuras capsulares y ligamentosas dorsales ${ }^{6}$. Los tornillos percutáneos de escafoides en fracturas agudas seleccionadas y en pacientes jóvenes son un avance en el período de curación, con reducción del tiempo de inmovilización, y con la reincorporación precoz a las tareas habituales y deportivas $^{4,8-11}$. En este estudio describimos nuestra experiencia con el uso de tornillos canulados percutáneos mini-Acutrak (Acumed, Hillsboro, OR) en las fracturas agudas de escafoides carpiano, de polo proximal, transversas y oblicuas de cuerpo sin desplazamiento, deformidades ni 

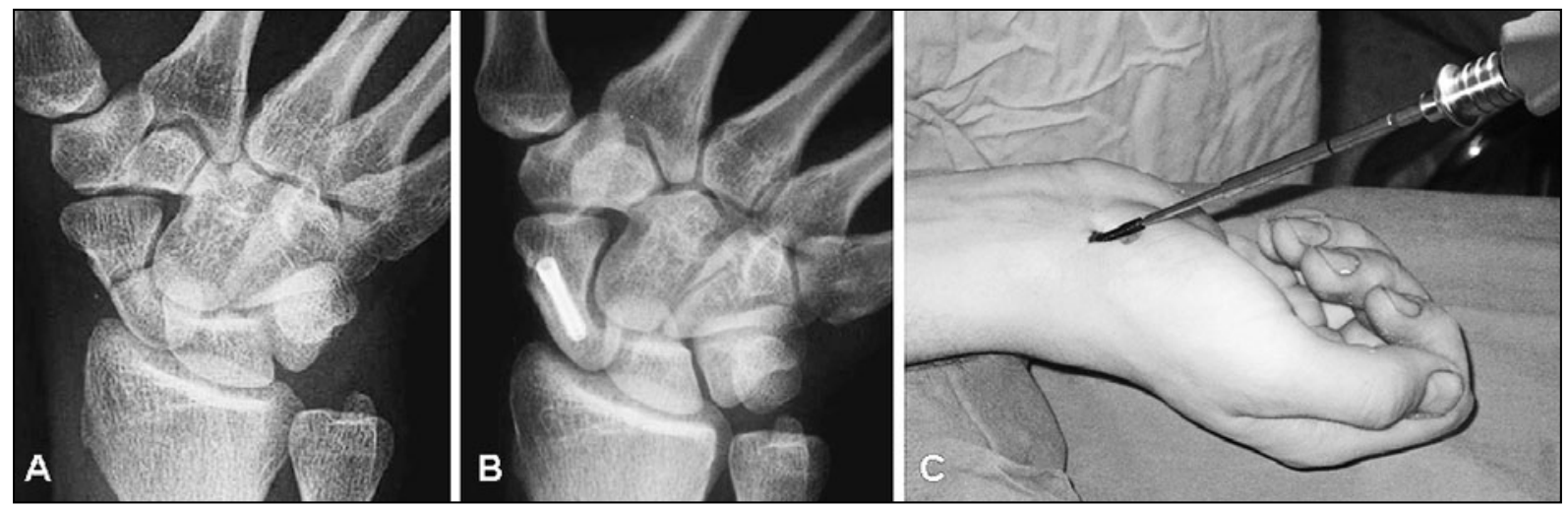

Figura 1. Abordaje palmar: a) Fractura no desplazada de cuerpo de escafoides, b) osteosíntesis en la proyección AP, c) Extensión de la muñeca para la introducción del tornillo.

inestabilidad carpiana. Discutimos las indicaciones, la técnica quirúrgica, y las posibles complicaciones.

\section{MATERIAL Y MÉTODO}

Desde noviembre de 2007 a septiembre de 2008 realizamos un estudio retrospectivo en 22 pacientes que fueron operados en la unidad de mano del Hospital de la Santa Creu i Sant Pau por lesiones traumáticas de escafoides. Fueron una mujer y 21 varones de edad media 27 años (rango: 18-47). El seguimiento medio fue de 6 meses (rango: 3-10). El dolor fue evaluado mediante la Escala Analógica Visual (de 0 a 10) antes de la intervención y en las visitas sucesivas. La movilidad de la muñeca fue medida con un goniómetro. Las proyecciones radiológicas incluyen la anteroposterior en posición neutra, desviación radial y desviación cubital, proyección lateral y oblicua con supinación de $20^{\circ}$. El tiempo transcurrido desde el traumatismo hasta la cirugía fue de 8 días de media (rango: 2-65), teniendo en cuenta que dos fracturas fueron tratadas inicialmente de forma conservadora. El patrón de fractura de escafoides se dividió según la clasificación de Herbert ${ }^{12}$. Sin embargo, debido a que esta clasificación incluye como inestable a toda fractura de cuerpo (Tipo B), hemos seguido los criterios de inestabilidad de las fracturas agudas de cuerpo de escafoides de la Clínica Mayo propuesta por Cooney et al. ${ }^{13}$. Esta última considera una fractura de cuerpo de escafoides inestable cuando el desplazamiento es mayor a $1 \mathrm{~mm}$, la angulación intraescafoidea lateral mayor de $35^{\circ}$, la pérdida o fragmentación ósea significativa, la fractura perisemilunar, la alineación en DISI, el ángulo escafolunar mayor a $60^{\circ} \mathrm{y} / \mathrm{o}$ el ángulo radio semilunar mayor a $15^{\circ}$. En nuestro estudio no incluimos fracturas de cuerpo de escafoides inestables.

Técnica quirúrgica: La colocación de la osteosíntesis percutánea depende del patrón de la fractura. La localización de la fractura es importante para decidir si el tornillo se coloca por vía palmar o por vía dorsal. Una fractura transversa estable de cuerpo de escafoides es ideal para una fijación con un tornillo percutáneo con dirección de palmar-distal a dorsal-proximal (Figura 1). Primero, se identifica el tubérculo del escafoides en la cara anterior de la muñeca con maniobras de desviación radial y cubital. El intensificador de imagen o el fluorescan se emplea en múltiples planos para asegurar que la aguja guía esté situada a lo largo del eje longitudinal del escafoides y que atraviese el foco de fractura. Para poder colocar la aguja guía se necesita unas tallas en el dorso de la muñeca que permitan hacer dorsiflexión. Con este gesto el trapecio se traslada dorsal y no está en el trayecto de la aguja. Si por el trazo de fractura se prevé que se puede desplazar la fractura al colocar la broca o el tornillo, se aconseja la colocación de una aguja paralela. La longitud del tornillo puede medirse indirectamente con una segunda aguja guía, o directamente con el medidor del instrumental. Esta caja lleva tornillos estándar, mini-Acutrak y micro-Acutrak. Es 

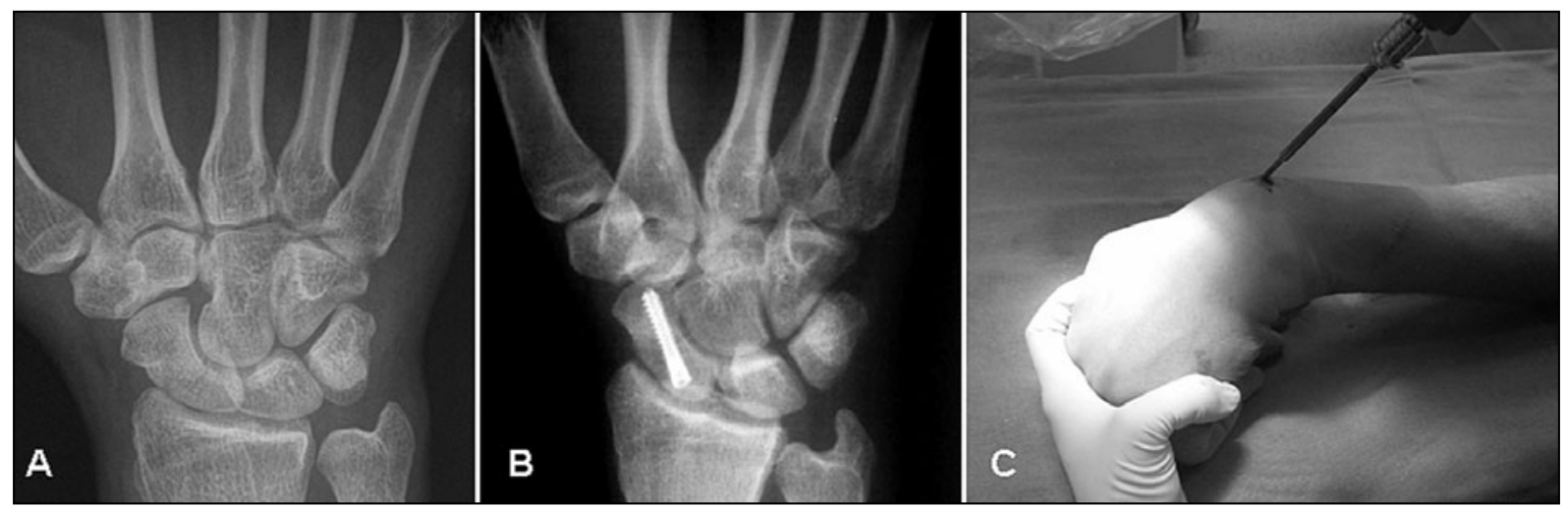

Figura 2. Abordaje dorsal: a) Fractura de polo proximal de escafoides. Osteosíntesis en la proyección AP, b) Flexión de la muñeca para la introducción del tornillo.

importante, no equivocarse de aguja guía y colocar el tornillo mini-Acutrak, que es el más adecuado para el escafoides. También es importante saber que, a pesar que parezca que mides correctamente, siempre se mide más de lo que debe ser, puesto que el tornillo comprime el foco de fractura y debe quedar completamente enterrado en el escafoides. Se recomienda restar a la medición entre 4-6 mm. Normalmente para el escafoides de un varón joven un tornillo de $18 \mathrm{~mm}$ es suficiente. Posteriormente se broca siguiendo la aguja guía y teniendo cuidado de no extraerla al retirar la broca. Se coloca el tornillo con visualización en distintas proyecciones. Al respecto de las agujas guía, hay que decir que son extremadamente delgadas y que no permiten maniobras bruscas puesto que se doblan con facilidad impidiendo brocar o ascender el tornillo. La aguja doblada suele romperse en el interior del escafoides o quedarse enganchada en la punta del tornillo, por lo que debería retirase. Una vez se ha colocado el tornillo percutáneo, se inmoviliza durante 10 días y posteriormente se inicia la fisioterapia. Esta técnica está contraindicada en caso de tratarse de una fractura de polo proximal u oblicua de cuerpo de escafoides, debido a que el tornillo no sería perpendicular a la línea de fractura y no podría hacer compresión. En estos casos el tornillo canulado se coloca por vía dorsal, con entrada proximal y cubital al tubérculo de Lister (Figura 2).

\section{RESULTADOS}

La osteosíntesis percutánea con tornillo canulado mini-Acutrak (Acumed, Hillsboro, OR) fue indicada en 15 fracturas mínimamente o no desplazadas de cuerpo escafoides (Herbert tipo


Figura 3. Consolidación radiológica de fractura de escafoides: a) proyección AP, b) proyección lateral, c) proyección oblicua 1, d) proyección oblicua 2. 

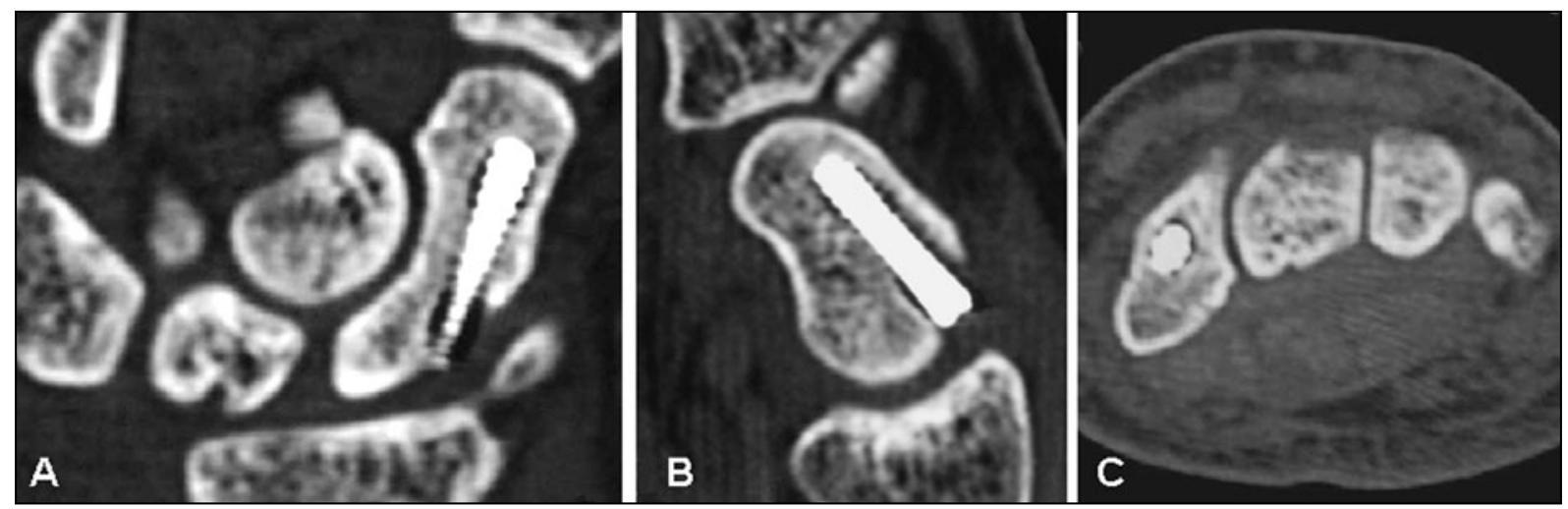

Figura 4. Consolidación de una fractura de cuerpo de escafoides tratada mediante técnica percutánea con tornillo mini-Acutrak: a) TAC corte coronal, b) TAC corte sagital, c) TAC corte axial.

A2 o B2), 3 fracturas oblicuas (Herbert tipo B1) y 4 de polo proximal (Herbert tipo B3). El mecanismo de fractura fue la caída con la muñeca en dorsiflexión, en 16 pacientes tras accidentes deportivos, en 3 por accidentes de tráfico con moto y en 3 casos caída casual. Según la escala analógica visual de dolor los pacientes pasaron de una puntuación media preoperatoria de 7 (rango: 5-9) a 1 (rango: 0-2) postoperatoria. $\mathrm{El}$ arco de movilidad al final del seguimiento fue completo en todos los pacientes comparado con la muñeca contralateral. La movilidad era casi igual a la contralateral a la segunda semana de retirar la inmovilización y de realizar fisioterapia, y oscilaba en $45-70^{\circ}$ de flexión y $65-$ $85^{\circ}$ de extensión. El grado de satisfacción final de los pacientes era bueno en el $100 \%$ de los casos, sin presentar dolor en reposo ni con las actividades de la vida diaria. Dos pacientes con entrada del tornillo percutáneo palmar, presen-

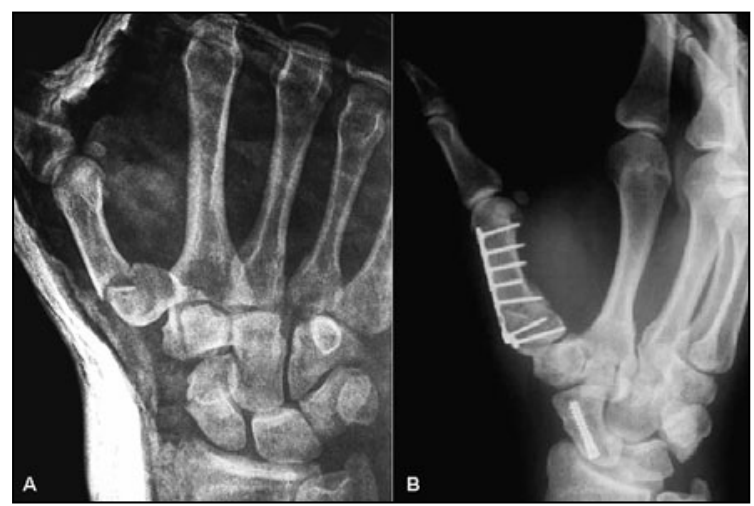

Figura 5. a) Fractura de cuerpo de escafoides asociada a fractura de Rolando. b) Osteosíntesis con placa de la fractura de Rolando y percutánea del escafoides. taban molestias con la hiperextensión de la muñeca. Todos los pacientes obtuvieron resultados buenos/excelentes con un tiempo de consolidación entre las 8 y 12 semanas (media de 6 semanas) (Figura 3). Se considera consolidación de la fractura cuando en las radiografías se aprecia una ausencia de línea de fractura o se observa paso de trabéculas óseas a través de la línea. La tomografía axial computerizada (TAC) fue útil en 8 casos para confirmar la consolidación del escafoides (Figura 4). La longitud media de los tornillos fue de 18mm (rango: 16-22). Hubo 3 casos de lesiones asociadas en la mis-

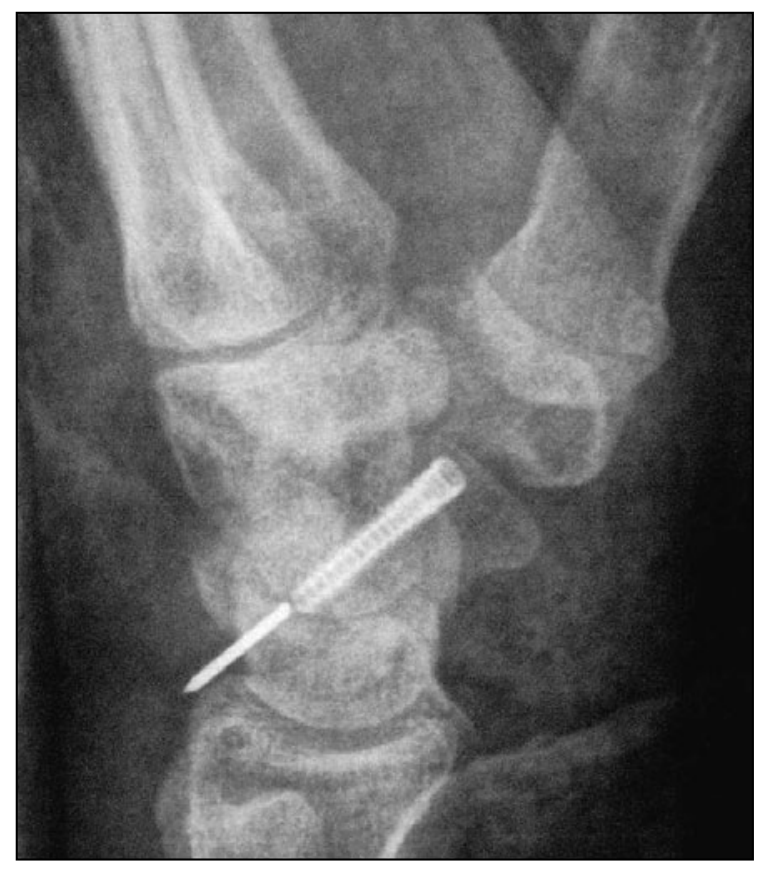

Figura 6. Ruptura de la aguja guía en el interior del tornillo. 
ma mano (Figura 5). La incorporación a la vida laboral y deportiva fue de media a las 7 semanas (rango: 4-12). Los 3 pacientes que se incorporaron a su actividad laboral a las 4 semanas no eran trabajadores de fuerza. Como complicación tuvimos un caso de ruptura de la aguja guía en el interior del tornillo. La migración dorsal de ésta produjo una tenosinovitis de extensores, con dolor al realizar fisioterapia que obligó a retirarla (Figura 6). Hemos tenido dos casos de restos de partículas metálicas por el roce de la broca corta con la aguja guía a la entrada del escafoides por vía palmar, y un caso de tornillo excéntrico sin repercusión clínica. No hemos apreciado casos de seudoartrosis, inestabilidad, infección, aflojamiento del tornillo o cambios degenerativos.

\section{DISCUSIÓN}

Las indicaciones de la osteosíntesis percutánea son las fracturas sin desplazar o con desplazamiento mínimo, transversas y oblicuas de cuerpo de escafoides y de polo proximal. Para el tratamiento de las fracturas desplazadas o con los criterios de inestabilidad de Cooney et al. ${ }^{13}$ se prefiere la reducción a cielo abierto ${ }^{1}$. La fijación percutánea es una alternativa razonable a la inmovilización con escayola para los pacientes con fracturas del escafoides no desplazadas o con desplazamiento mínimo, excepto en niños y ancianos ${ }^{11,14}$. Se utiliza un abordaje dorsal o palmar según el trazo de fractura ${ }^{11,14}$. El implante debe colocarse en una situación óptima. Para ello, la aguja guía debe colocarse a través del eje axial central del escafoides con la fractura reducida ${ }^{11}$. Por estudios biomecánicos se ha demostrado que los tornillos de escafoides centrados son un sistema más rígido que los colocados excéntricos ${ }^{2,3,11}$. En el estudio anatómico de Chan et al. ${ }^{15}$ se observa que el abordaje percutáneo dorsal en las fracturas de cuerpo permite una colocación más centrada del tornillo en el polo distal, pero no existen diferencias respecto del abordaje palmar cuando se valoran otras áreas como el polo proximal o el cuerpo.

La osteosíntesis percutánea de las fracturas de escafoides no desplazadas o mínimamente desplazadas consigue cerca del $100 \%$ de conso- lidación con complicaciones mínimas². Según Moser et al..$^{5}$ la osteosíntesis percutánea de 17 fracturas de escafoides agudas tiene un porcentaje de consolidación del 100\%. Sin embargo, en una serie más amplia de 68 casos se observaron dos seudoartrosis atribuidas a errores técnicos. En nuestra serie de 22 casos hemos apreciado la consolidación de la fractura en todos los casos en un tiempo medio de 6 semanas. Una de las limitaciones de estos estudios es poder asegurar la consolidación, por lo que nosotros en caso de dudas con la radiografía solicitamos una TAC que confirme la unión. La incorporación de los tornillos percutáneos de escafoides ha permitido una ampliación del tratamiento quirúrgico a fracturas tratadas habitualmente con yeso en pacientes jóvenes reduciendo los casos de retardo de consolidación, seudoartrosis o necrosis avascular ${ }^{8}$. Se han descrito como complicaciones, la pérdida de reducción, la protusión del tornillo, la lesión de partes blandas y la incorrecta colocación de la osteosíntesis ${ }^{2}$. Los fallos más comunes son la selección de un tornillo demasiado largo o su colocación excéntrica. La incidencia de colocación de tornillos demasiado largos en la bibliografía es del $1,5 \%{ }^{11}$. En nuestra serie no hemos tenido ningún caso de tornillo largo, pero si un caso colocación excéntrica sin repercusión clínica y con consolidación de la fractura. Algunas de las complicaciones intraoperatorias $\operatorname{son}^{4,8}:$ 1) El desplazamiento de la fractura cuando se introduce el tornillo. Esto suele ocurrir en las fracturas de polo proximal y en las oblicuas. Se debe a una inadecuada colocación de la aguja guía o de la reducción de la fractura; 2) Colocar el tornillo con la fractura mal reducida; 3) Brocar en exceso puede dar lugar a un aflojamiento del tornillo y brocar poco puede desplazar la fractura o impedir la adecuada colocación de la osteosíntesis; y 4) Riesgo de lesión de la arteria radial, aunque este es mínimo. Por estudios anatómicos se ha demostrado que la aguja guía dentro del escafoides y colocada desde el tubérculo se sitúa a $14 \mathrm{~mm}$ de la arteria radial, a $19 \mathrm{~mm}$ de la rama superficial del nervio radial, y a $5 \mathrm{~mm}$ de la rama superficial de la arteria radial ${ }^{16}$. Nosotros no hemos tenido complicaciones vasculo-nerviosas.

La técnica percutánea es sencilla, pero requiere de una curva de aprendizaje y de una in- 
dicación correcta. Hay que tener en cuenta que no se deben realizar cambios bruscos de dirección de la aguja guía mientras se está colocando puesto que ésta puede doblarse o romperse impidiendo la correcta colocación del tornillo, como ocurrió en uno de nuestros pacientes y co- mo está descrito en la literatura ${ }^{5,17}$. En vista de nuestros resultados creemos que los tornillos percutáneos de escafoides mini-Acutrak aplicados en fracturas agudas seleccionadas y en pacientes jóvenes permiten la consolidación de la fractura con un menor tiempo de inmovilización.

\section{BIBLIOGRAFÍA}

1. Retting AC. Management of acute scaphoid fractures. Hand Clin 2000; 16: 381-95.

2. Gutow AP. Percutaneous fixation of scaphoid fractures. J Am Acad Orthop Surg 2007; 15: 474-85.

3. Slade JF, Dodds SD. Minimally invasive management of scaphoid nonunions. Clin Orthop 2006; 445: 108-19.

4. Bond CD, Shin AY. Percutaneous cannulated screw fixation of acute scaphoid fractures. Tech Hand Upper Extrem Surg 2000; 4: 81-7.

5. Moser VL, Krimmer H, Herbert TJ. Minimal invasive treatment for scaphoid fractures using the cannulated Herbert screw system. Tech Hand Upper Extrem Surg 2003; 7: 141-6.

6. Haddad FS, Goddard NJ. Acutrak percutaneous scaphoid fixation. Tech Hand Upper Extrem Surg 2000; 4: 78-80.

7. Muzaffar AR, Carter PR. Vascularized bone grafting and Herbert screw fixation of scaphoid nonunions with avascular proximal poles. Tech Hand Upper Extrem Surg 2002; 6: 155-64.

8. Shin AY, Hofmeister EP. Percutaneous fixation of stable scaphoid fractures. Tech Hand Up Extrem Surg 2004; 8: 87-94.

9. González del Pino J. Comentario al artículo de López de la Garma F y Labreda M. Fracturas del escafoides carpiano. Rev Ortop Traumatol 2005; 49: 83-9.

10. Sanjuán R, Guillen M, García de Lucas F. Osteosíntesis percutánea de las fracturas de escafoides. Rev lberamer Cir Mano 2006; 34: 33-40.

11. Slade JF, Moore AE. Dorsal percutaneous fixation of stable, unstable, and displaced scaphoid fractures and selected nonunions. Hand Clin 2003; 8: $1-18$.

12. Herbert TJ. The fractured scaphoid. St Louis, MO: Quality Medical Publishing, 1990.

13. Cooney WP, Dobyns JH, Linscheid RL. Fractures of the sca- phoid: A rational approach to management. Clin Orthop 1980; 149: 90-7.

14. Cooney WP. Fracturas del escafoides: tratamientos y técnicas actuales. En: Traumatismos, Miembro superior. Paul Tornetta III. Instructional Course Lectures. Americam Academy of Orthopaedic Surgeons. Edición en español. Barcelona: Editorial Médica Paramericana, 2008: 85-96.

15. Chan K.W. Mc Adams T.R. Central screw placement in percutaneous screw scaphoid fixation: a cadaveric comparison of proximal and distal techniques. J Hand Surg Am 2004; 29: 74-9.

16. Kamineni S, Lavy CB. Percutaneous fixation of scaphoid fractures: an anatomic study. J Hand Surg Br 1999; 24: 85-8.

17. Heras Palou C. Aspectos técnicos de la fijación percutánea de las fracturas de escafoides. Rev Iberamer Cir Mano 2006; 34: 27-32. 